\title{
Cognitive impairment and CSF proteome modification after oral bacteriotherapy in HIV patients
}

\author{
Claudia Landi ${ }^{1}$ - Letizia Santinelli ${ }^{2}$. Laura Bianchi ${ }^{1}$ - Enxhi Shaba ${ }^{1}$. Giancarlo Ceccarelli ${ }^{3}$ (D) \\ Eugenio Nelson Cavallari ${ }^{3}$. Cristian Borrazzo ${ }^{3}$. Claudia Pinacchio ${ }^{3} \cdot$ Carolina Scagnolari $^{2} \cdot$ Vincenzo Vullo $^{3}$. \\ Luca Bini $^{1} \cdot$ Gabriella d'Ettorre ${ }^{3}$
}

Received: 6 May 2019 / Revised: 22 August 2019 / Accepted: 27 August 2019

(C) Journal of NeuroVirology, Inc. 2019

\begin{abstract}
Objective: To investigate whether a probiotic supplementation to cART patients modifies the cerebrospinal fluid (CSF) proteome and improves neurocognitive impairment. Methods: 26 CSF samples from 13 HIV-positive patients [six patients living with HIV (PLHIV) and seven patients with a history of AIDS (PHAIDS)] were analyzed. All patients underwent to neurocognitive evaluation and blood sampling at baseline and after 6 months of oral bacteriotherapy. Immune phenotyping and activation markers (CD38 and HLA-DR) were evaluated on peripheral blood mononuclear cells (PBMC). Plasma levels of IL-6, sCD14, and MIP-1 $\beta$ were detected, by enzyme-linked immunosorbent assay (ELISA). Functional proteomic analysis of CSF sample was conducted by two-dimensional electrophoresis; a multivariate analysis was performed by principal component analysis (PCA) and data were enriched by STRING software. Results: Oral bacteriotherapy leads to an improvement on several cognitive test and neurocognitive performance in both groups of HIV-positive subjects. A reduction in the percentage of CD $4^{+} \mathrm{CD} 38^{+} \mathrm{HLA}-\mathrm{DR}{ }^{+} \mathrm{T}$ cells was also observed at peripheral level after the probiotic intake $(p=0.008)$. In addition, the probiotic supplementation to cART significantly modifies protein species composition and abundance at the CSF level, especially those related to inflammation ( $\beta 2$-microglobulin $p=0.03$; haptoglobin $p=0.06$; albumin $p=0.003$; hemoglobin $p=0.003$; immunoglobulin heavy chains constant region $p=0.02$, transthyretin $p=0.02$ ) in PLHIV and PHAIDS. Conclusions: Our results suggest that oral bacteriotherapy as a supplement to cART could exert a role in the amelioration of inflammation state at peripheral and CNS level.
\end{abstract}

Keywords HIV · Probiotic · Functional proteomics · Immunoactivation · Bacteriotherapy

\section{Introduction}

Notwithstanding the antiretroviral treatment, most HIV-infected individuals show a high prevalence of non-AIDS defining neurologic conditions collected under the name of HIV-associated neurocognitive disorders (HAND) (Heaton et al. 2010, 2011). Neuroinflammation seems to be aggravated by bacterial products leaking out of the colon into the bloodstream and causing a greater level of inflammation throughout the body (Vera et al.

Giancarlo Ceccarelli

giancarlo.ceccarelli@uniroma1.it

1 Laboratory Functional Proteomics, Department Life Sciences, University of Siena, Siena, Italy

2 Department of Molecular Medicine, Laboratory of Virology, Sapienza University of Rome, Rome, Italy

3 Department of Public Health and Infectious Diseases, Sapienza University of Rome, Viale del Policlinico 155, 00161 Rome, Italy
2015, 2016; Spudich et al. 2005, 2011; Banks et al. 2015; Gold et al. 2014; Cinque et al. 2007). Since imbalances of gut bacterial species (dysbiosis) and increased microbial translocation have been observed in HIV-positive patients (Xu et al. 2013; Salas and Chang 2014; Hunt et al. 2014), any intervention which is able to protect the gut barrier should be explored. Previously, we have shown that the supplementation with a high concentration multistrain probiotic formulation to cART patients was associated with the recovery of the gut epithelial barrier integrity, a reduction in both intraepithelial lymphocyte density and enterocyte apoptosis, and an improvement in mitochondrial morphology (d'Ettorre et al. 2014, 2017; Carter et al. 2016; D'Angelo et al. 2017; Pinacchio et al. 2018). We also observed that different neurocognitive test scores improved significantly after probiotic supplementation (Ceccarelli et al. 2017a, b; Akbari et al. 2016).

Nevertheless, our hypothesis was that HIV directly impairs the neurological system, independently from the years of exposure to the virus, the disease's severity and the 
immunological status of patient. Given these considerations, the scope of our present research was to investigate if, according to a previous diagnosis of AIDS, there were differentially abundant protein species associated with the HIV-related neurocognitive impairment.

Moreover, we wanted to investigate if a modification of the cerebrospinal fluid (CSF) proteome occurs in cART patients after oral bacteriotherapy, in order to confirm the previously observed gut and neurological improvements at the molecular level.

\section{Materials and methods}

\section{Study population}

In this longitudinal interventional non-randomized study, we included 13 eligible HIV-positive subjects: six patients living with HIV (PLHIV) and seven patients with a history of AIDS (PHAIDS) referring for routine visits to the HIV Outpatient Clinic of the Department of Infectious Diseases of "Sapienza" University of Rome. The inclusion criteria were (1) HIVpositive patients who had signed the informed consent, (2) women or men at least 18 years of age, (3) in cART with HIV-RNA $<50$ copies $/ \mathrm{ml}$, and CD4 counts $>400$ cells $/ \mathrm{mm}^{3}$.

Exclusion criteria were (1) known allergy or intolerance to the product, (2) diarrhea, (3) history of or current inflammatory diseases of the small or large intestine, (4) any past or current systemic malignancy, (5) previous or actual drug addiction, (6) use of antibiotics or probiotics during the 3 weeks prior the enrollment, (7) previous or actual psychiatric disorders, (8) previous or actual neurological disorders, (9) positive PCR on CSF for any of the following pathogens: CMV, EBV, HSV1, HSV2, VZV, HHV8, BKV, JCV, pregnancy.

Because lumbar puncture is considered a procedure with several risks and in literature there is no reported study where lumbar puncture was performed in the control arm, our institution considered it unethical to have a control group undergoing such a procedure.

\section{Standard protocol approvals, registration, and patients' consents}

The study was approved by the internal committee of the Infectious Diseases Department and by the Ethics Committee of "Sapienza" University of Rome. A written informed consent was obtained from all patients prior to enrollment.

\section{Study timeline and investigational compound}

All participants underwent cerebrospinal fluid (CSF) sampling and neurocognitive evaluation at baseline (T0) and after 6 months (T6) of supplementation with a commercially available multistrain probiotic formulation (trade name: Vivomixx ${ }^{\circledR}$ in EU and Visbiome ${ }^{\circledR}$ in USA; composition: Streptococcus thermophilus DSM24731, Bifidobacterium breve DSM24732, Bifidobacterium longum DSM24736, Bifidobacterium infantis DSM24737, Lactobacillus acidophilus DSM24735, Lactobacillus plantarum DSM24730, Lactobacillus paracasei DSM24733, Lactobacillus delbrueckii ssp. bulgaricus DSM24734).

\section{Specimen processing}

About $20 \mathrm{~mL}$ of whole blood was collected by venipuncture in Vacutainer tubes containing EDTA (BD Biosciences, San Jose, CA, USA) at each study visit. Peripheral blood mononuclear cells were separated by Ficoll gradient centrifugation (Lympholyte; Cedarlane Labs, Hornby, ON, Canada) and washed twice in phosphate-buffered saline solution (Ceccarelli et al. 2017b). Freshly isolated PBMCs were used immediately for immune phenotyping and activation staining. About $10 \mathrm{~mL}$ of whole blood was collected by BD Vacutainer Plus Plastic Serum. After centrifuge, serum was stored in aliquots at $-80{ }^{\circ} \mathrm{C}$.

\section{T cell phenotyping by flow cytometry}

Phenotypes and activation markers were evaluated by Miltenyi Biotec flow cytometer-MACSQuant Analyzer (eight fluorescence channels, three lasers) on freshly isolated PBMC. Immune activation was evaluated by multiparameter flow cytofluorimetric analysis by the following antihuman monoclonal antibodies: CD3-PerCP, CD4-APC-Vio770, CD38-APC, and HLA-DR-PE (Miltenyi Biotec, Bergisch Gladbach, Germany).

\section{Enzyme-linked immunosorbent assay}

Plasma levels of IL-6, sCD14, and MIP- $1 \beta$ were detected by enzyme-linked immunosorbent assay (ELISA) kit (R\&D System, MN, USA) based on an indirect assay with an antigen-antiserum bound and a conjugate antibody using a 3,3',5, 5'-tetramethylbenzidine (TMB) as a substrate for the detection. For cytokine detection, we used an ELISA assay with an anti-IL6, sCD14, and MIP-1 $\beta$ antibody precoated microplate; a cytokines antiserum; a conjugate enzyme (goat antirabbit immunoglobulins conjugated with peroxidase); and a substrate for the reaction (containing tetramethylbenzidine, substrate buffer, and hydrogen peroxide). The reaction was monitored at $450 \mathrm{~nm}$. Quantification was achieved by comparing their absorbance with a reference curve (prepared with known standard concentrations). 


\section{Virologic analysis}

HIV-1 RNA copy number was measured in plasma by using Versant kPCR (Siemens Healthcare Diagnostic, Inc., Tarrytown, NY). The detection limit was 37 HIV-1 RNA copies $/ \mathrm{mL}$.

\section{Neuropsychological investigation}

Neuropsychological tests were performed by a trained neuropsychologist in order to explore patients' ability in the following areas: verbal, language, attention, learning memory, working memory, abstraction, executive functions, processing speed of information (sensory-perceptual and motor). The test battery included Rey-Osterrieth Complex Figure Test (ROCF) to evaluate participants' recognition and recall skills for non-verbal contents; Rey Auditory Verbal Learning Test (RAVLT) to evaluate short-term auditory verbal memory, rate of learning, and retention of information; test of weights and measures estimation (STEP) to evaluate abstraction skills; visual search test (attention matrices test) to evaluate attention skills; verbal fluency test (FAB) to evaluate executive functions and the ability to switch between different tasks; test of phonological and semantic verbal fluency (respectively PVF and SVF) to evaluate phonological and semantic supplies and the ability to access them; Raven's Standard Progressive Matrices (SPM) to evaluate abstract reasoning and problem solving capabilities; digit span test to evaluate short-term memory and executive functions; Corsi block-tapping test (CBTT) to evaluate short-term spatial memory and executive functions; Aachener Aphasia Test (AAT) to evaluate the presence of aphasia among study participants; and trail making test A and B (TMT A and TMT B) to evaluate visual-spatial attention and motor skills. To minimize the possible influence of the practical effect on neuropsychological performances, parallel/alternate forms were used.

Moreover, effect sizes were also extracted for descriptive purposes as well as baseline and post-concussion means and standard deviations for the $\mathrm{T} 0$ and $\mathrm{T} 6$.

\section{Functional proteomic analysis of CSF by two-dimensional electrophoresis}

Differential proteomic analysis was conducted on $\mathrm{T} 0$ and $\mathrm{T} 6$ CSF samples of participants. Sample preparation was performed as reported: $333 \mu \mathrm{l}$ of CSF was precipitated 1:4 in cold acetone and centrifuged at $10,000 \times g$ for $15 \mathrm{~min}$. The supernatant was eliminated and the pellet solubilized in $5 \mu \mathrm{l}$ of $5 \%$ $\mathrm{w} / \mathrm{v}$ SDS and $2.3 \% \mathrm{w} / \mathrm{v}$ DTE and heated at $95{ }^{\circ} \mathrm{C}$ for $7 \mathrm{~min}$. Rehydration buffer (8 M UREA, 2 M Thiourea, $1 \%$ w/v DTE, $0.4 \% \mathrm{w} / \mathrm{v}$ CHAPS, and traces of bromophenol blue) was, then, added to reach a total volume of $450 \mu \mathrm{l}$, containing $60 \mu \mathrm{g}$ of total proteins. Preparative gels were processed as the previous ones, starting from $3 \mathrm{ml}$ of CSF to obtain a final protein load of $540 \mu \mathrm{g}$.

CSF samples were resolved by two-dimensional electrophoresis (2DE), as reported (Hochstrasser et al. 1988). Spots were considered differentially abundant between two conditions when the ratio of the percentage of relative volume $(\% \mathrm{~V}$ : Vol of a single spot divided by the total volume of spots computed over the whole image and expressed in percentage) means was greater than twofold. Statistical non-parametric Mann-Whitney test $(p \leq 0.05)$, by IBM SPSS $®$ software platform, was performed to determine if two sets of data were significantly different from each other. $\% \mathrm{~V}$ of differentially abundant spots was used to perform multivariate analysis by principal component analysis (PCA) simplifying the amount of data by linear transformation. PCA enables us to visualize experimental groups on a two-dimensional plane on the basis of the variable spot patterns. Spots of interest were identified by peptide mass fingerprinting (PMF) on an ultrafleXtreme ${ }^{\mathrm{TM}}$ MALDI-ToF/ToF instrument (Bruker Corporation, Billerica, MA, USA). Identified protein species were used to perform functional and pathways analysis by STRING software.

\section{Statistical analysis}

Data are expressed as median/IQR unless otherwise indicated. The demographic and clinical characteristics between PLHIV and PHAIDS were compared using Student's $t$ and chisquared tests. Differences in the neurological test scores between T0 and T6 were evaluated using Student's $t$ test. Differences in the levels of CD4 T cells expressing CD38 and HLA-DR markers in HIV-1-infected patients at T0 and T6 were evaluated using the Wilcoxon test for paired samples. The same test was used to compare the levels of protein species identified by mass spectrometry between $\mathrm{T} 0$ and $\mathrm{T} 6$. Differences were considered statistically significant when $p$ value were less of 0.05 . All analyses were performed with the SPSS v.20.0 for Windows (IBM, New York, NY, USA).

\section{Results}

\section{Demographic and clinical characteristics of HIV-1-infected participants}

In the present study, 13 HIV-1-infected subjects were enrolled: six were patients living with HIV (PLHIV) and seven patients with a condition of AIDS (PHAIDS). All patients had a mean age of 45.5 years and were virologically suppressed (HIVRNA $<37$ copies $/ \mathrm{mL}$ ). The mean CD4+ T cell count was 669.25 (IQR 493-1080) and the median duration of treatment was 10.75 years (IQR 7.75-16.25). At enrolment, median Neopterin levels were $41.36 \mathrm{nmol} / \mathrm{L}$ (IQR 31.39-70.43) for PLHIV group and $43.82 \mathrm{nmol} / \mathrm{L}$ (IQR 21.5-56). No 
Table 1 Neurocognitive evaluation

Neurocognitive tests

Rey-Osterrieth Complex Figure Test (ROCF) immediate recall

$16.4 \pm 4.01$ vs $21.6 \pm 3.1$

$(p=0.002)$

Rey-Osterrieth Complex Figure Test (ROCF) delayed recall

Rey Auditory Verbal Learning Test (RAVLT) immediate recall

Rey Auditory Verbal Learning Test (RAVLT) delayed recall

Rey Auditory Verbal Learning Test (RAVLT) recognition

Test of weights and measures estimation (STEP) — time

Test of weights and measures estimation (STEP) — weights

Test of weights and measures estimation (STEP) - total

Visual search test (attention matrices test)

Verbal fluency test (FAB)

Test of phonological verbal fluency (PVF)

Test of semantic verbal fluency (SVF)

Raven's Standard Progressive Matrices (SPM)

Verbal span test forward

Verbal span test backward

Corsi block-tapping test (CBTT) forward

Corsi block-tapping test (CBTT) backward

Aachener Aphasia Test (AAT)

Trail making test A (TMT A)

Trail making test B (TMT B)
$15.5 \pm 3.8$ vs $23.2 \pm 2.6$

$(p=0.001)$

$40.8 \pm 10.4$ vs $52.2 \pm 3.9$

$(p=0.019)$

$7.83 \pm 2.9$ vs $11.9 \pm 2.3$

$(p=0.024)$

$94.5 \pm 4.7$ vs $98.5 \pm 1.6$

$(p=0.131)$

$17.6 \pm 4.9$ vs $22.7 \pm 1.6$

$(p=0.027)$

$17.3 \pm 3.5$ vs $21.7 \pm 1.7$

$(p=0.03)$

$36.6 \pm 7.6$ vs $44.2 \pm 2.9$

$(p=0.06)$

$49.3 \pm 7.5$ vs $48.1 \pm 2.8$

$(p=0.46)$

$14.2 \pm 1.8$ vs $15.7 \pm 1.9$

$(p=0.23)$

$29.8 \pm 9.4$ vs $42.8 \pm 3.6$

$(p=0.014)$

$45.3 \pm 10.8$ vs $47.8 \pm 1.8$

$(p=0.708)$

$28.3 \pm 5.6$ vs $30.2 \pm 3.8$

$(p=0.14)$

$4.5 \pm 1.0$ vs $5.3 \pm 0.8$

$(p=0.153)$

$4.4 \pm 0.7$ vs $4.5 \pm 0.7$

$(p=1.0)$

$4.51 \pm 0.6$ vs $5.24 \pm 0.4$

$(p=0.05)$

$3.69 \pm 0.7$ vs $4.1 \pm 0.6$

$p=0.19$

$9 \pm 0$ vs $9 \pm 0$

$(p=1.00)$

$52.1 \pm 11.7$ vs $45.5 \pm 7$

$(p=0.10)$

$132.3 \pm 43.9$ vs $107.8 \pm 34.5$

$(p=0.42)$
$1.4(0.59-2.32)$

$1.7(0.80-2.70)$

$1.58(0.7-2.46)$

$1.53(0.65-2.4)$

$1.14(0.31-1.96)$

$1.58(0.70-2.46)$

$1.39(0.53-2.24)$

$1.32(0.47-2.16)$

$-0.23(-1.0-0.54)$

$0.81(0.01-1.61)$

$1.80(0.89-2.72)$

$0.35(-0.42-1.13)$

$0.38(-0.37-1.17)$

$-0.14(-0.91-0.63)$

$0.14(-0.6-0.91)$

$1.43(0.53-2.29)$

$0.62(-0.16-1.41)$

N.A.

$0.73(-0.07-1.51)$

$0.62(-0.16-1.41)$

Differences in neurological test scores between T0 and T6 were evaluated using Student's $t$ test

${ }^{\mathrm{a}}$ Data are expressed as mean $\pm \mathrm{SD}$

statistically significant differences were recorded between the two groups at enrolment.

\section{Neurocognitive evaluation}

Participants were evaluated by a neuropsychologist before and after supplementation with oral probiotics. At T0, all subjects showed abnormal results in at least one test evaluating executive function and most presented a pathological impairment in two different domains. No significant differences were observed between PLHIV and PLAIDS. As shown in Table 1, after oral bacteriotherapy supplementation, all participants showed improvement in several neurocognitive tests and at T6, all investigated subjects showed normal neurocognitive performance.

\section{Peripheral immune activation}

We compared the immune activation of PBMCs measuring the levels of CD4 T cells expressing CD38 and HLA-DR markers at $\mathrm{T} 0$ and after bacteriotherapy supplementation (T6). Results showed a statistically significant reduction in the percentage of $\mathrm{CD} 4^{+} \mathrm{CD} 38^{+} \mathrm{HLA}^{-} \mathrm{DR}^{+} \mathrm{T}$ cells at $\mathrm{T} 6$ (median 0.18 [IQR 0.04-0.32]) compared with T0 (median 0.80 [IQR 0.37-2.16]); $p=0.008$ in both groups of patients. 


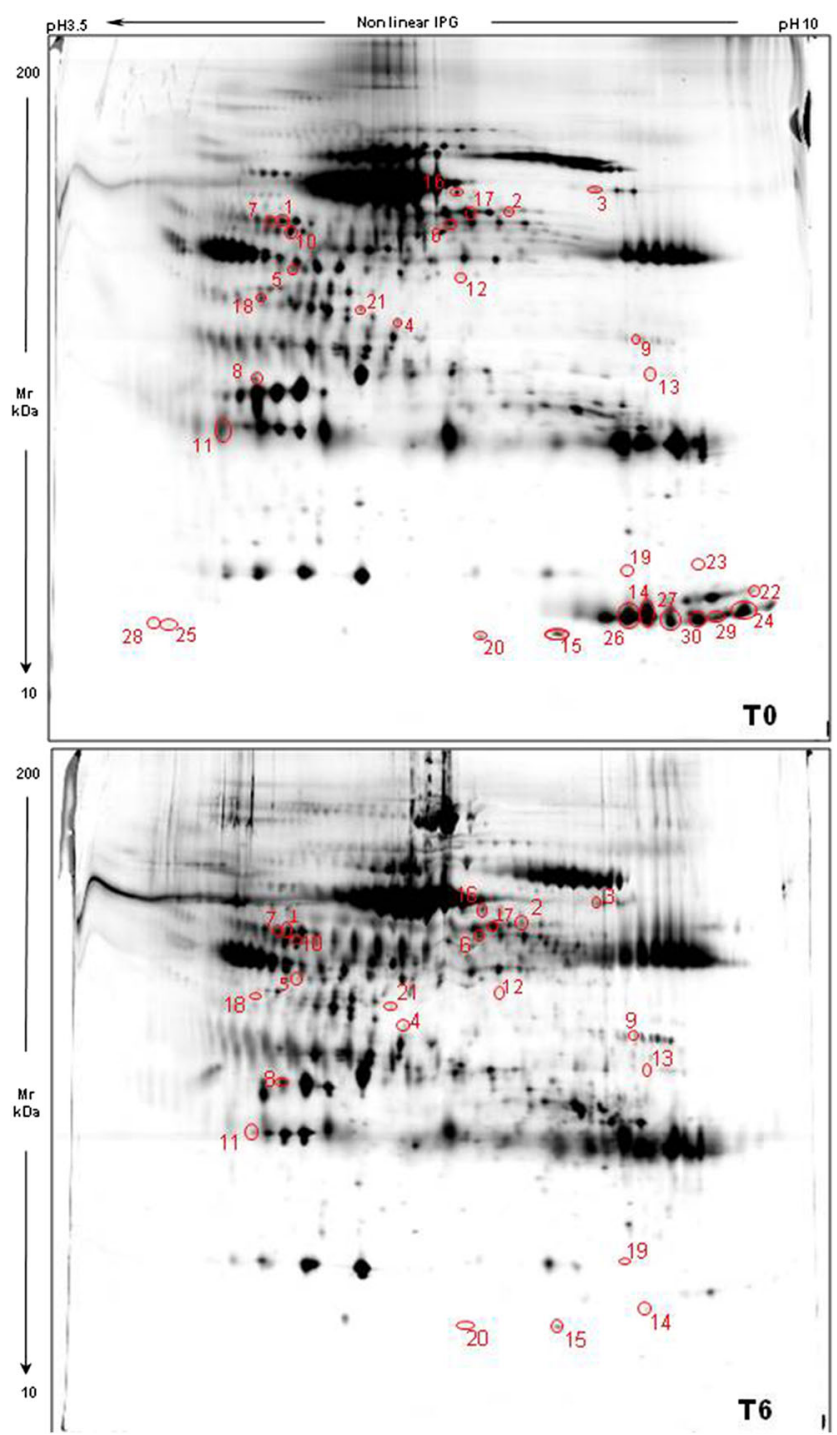

Fig. 1 Gel masters from T0 and T6 conditions. Differentially abundant spots are reported by red circles and numbers referred to those reported in Table 2

\section{Enzyme-linked immunosorbent assay}

In order to evaluate the status of inflammation before and after oral bacteriotherapy supplementation, we analyzed plasma levels of IL-6, sCD14, and MIP-1 $\beta$ at T0 and T6. Our results showed no significant differences in cytokine levels before [IL-6 (median 1.32; IQR 0.89-2.42); sCD14(median 1832.65; IQR 1520.882018.13); MIP-1 $\beta$ (median 46.47; IQR 26.81-53.23)] or after probiotic intake [IL-6 (median 1.75; IQR 0.7-3.13); sCD14(median 1781.85; IQR 1397.72-2038.72); MIP-1 $\beta$ (median 27.65; IQR 18.32-52.03)] ( $P>0.05)$.

\section{Differential proteomic analysis}

Image analysis highlighted 30 differentially abundant spots between T0 and T6 (Fig. 1). In addition, mass spectrometry analysis unambiguously identified 23 protein species (Table 2). Interestingly, most of the protein species showed a decreased amount in T6 samples, including spots 14, 22, 24, 26, 27, 29, 30 (Fig. 2), identified as hemoglobin $\alpha$ and $\beta$. Many of the remaining proteins could be directly related to inflammation as positive or negative reactants ( $\beta 2$-microglobulin $p=0.03$; haptoglobin $p=0.06$; albumin $p=0.003$; hemoglobin $p=0.003$; immunoglobulin heavy chains constant region $p=0.02$, transthyretin $p=0.02$ ) (Bini et al. 1992, 1996).

\section{Principal component analysis}

Principal component analysis (PCA) is a multivariate analysis that correlates a high number of variables $(\% \mathrm{~V})$ in order to evaluate whether the differential protein spots found were related to the analyzed conditions. Two Cartesian systems are reported in Fig. 3. The graph in Fig. 3a reports the 2D space where variables with the highest variance were projected on the first axis (PC1) with a variance of $36.2 \%$, while variables with the second highest variance (20.4\%) were reported on the second axis (PC2). This is easy to observe since the distribution of each sample relies on the differential abundance of spots in CSF (spot maps). Interestingly, six out of ten T0 samples were well distinguished from T6 samples on both the PC1 and PC2 axis. Conversely, the four T0 samples that were grouped together with $\mathrm{T} 6$ were well separated in a second PC analysis visualizing the PC1 (36.2\%) vs the PC3 (16.9\%) axis (Fig. 3b). This suggests an independent distribution of those four T0 samples (circled in green) against the T6 ones. The particular distribution of the spot maps inside the two groups is noteworthy: at $\mathrm{T} 0$, the distance correlation is high, and the spot maps are mainly grouped in two areas while T6 samples showed a lower distance correlation and were distributed in a well-defined area.

\section{Cluster analysis}

After protein identification, a cluster analysis was performed as shown in Fig. 4 in order to visualize how the differential protein species pattern in CSF behaved between $\mathrm{T} 0$ and $\mathrm{T} 6$. The $\% \mathrm{~V}$ mean normalized values of the 30 differentially abundant spots in T0 and T6 were organized in a hierarchical cluster. The Euclidean distance used gave us a hierarchical tree subdividing the protein spots in two major clusters on the basis of their different abundances. The first cluster holds two spots $(8,11)$ with an increased abundance in T6 and corresponding to transthyretin. The second cluster could be subdivided in two sub-clusters: the first one (spots 2, 1, 21, $13,6,19,3,5,7,12,16,4,15,18,17,10,9,20)$ showed a moderate decrease of protein abundance from T0 to T6 and the second represents all the protein species (spots 14, 22, 23, $24,25,26,27,28,29,30$ ) showing a strong decease at $\mathrm{T} 6$. 


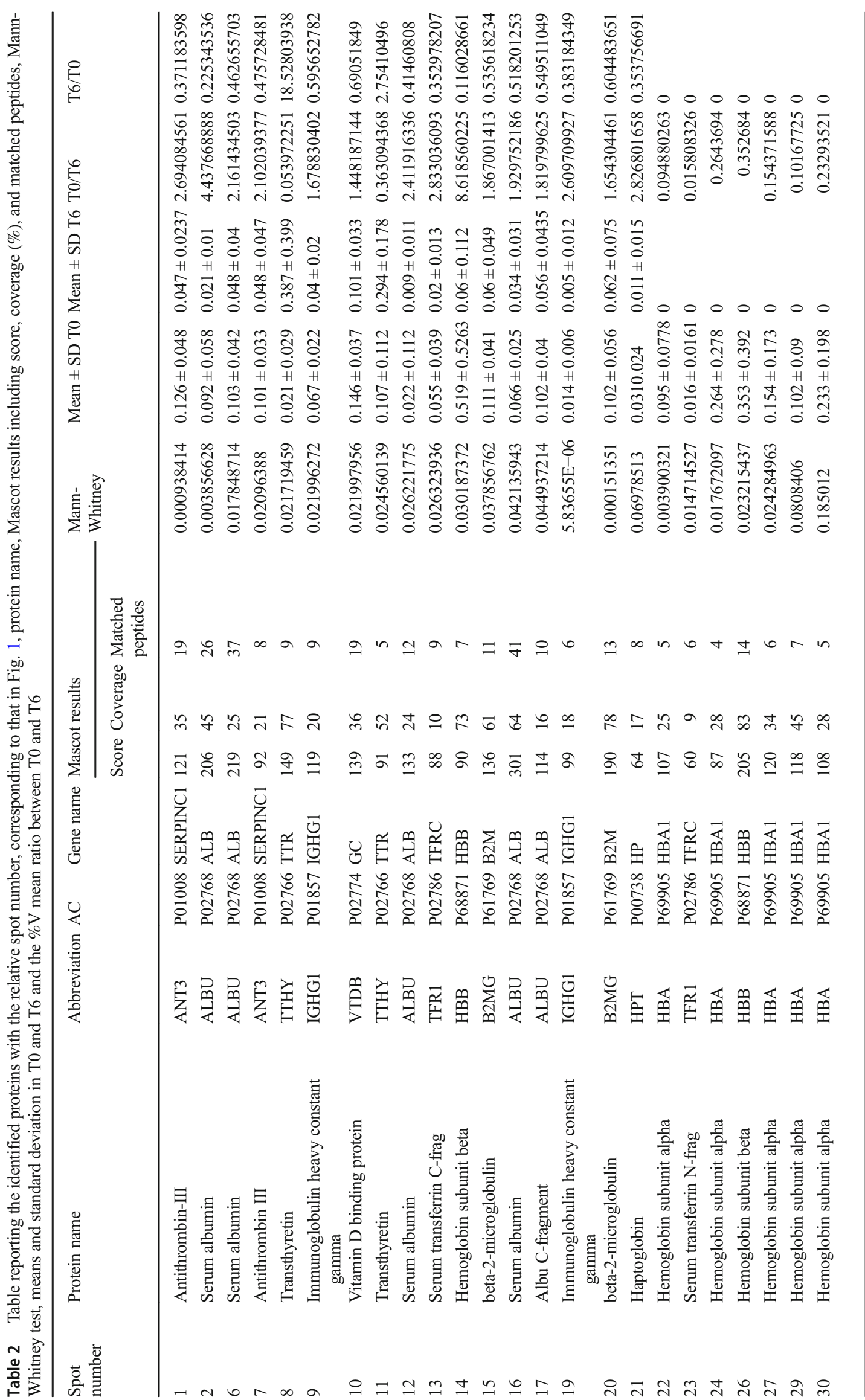




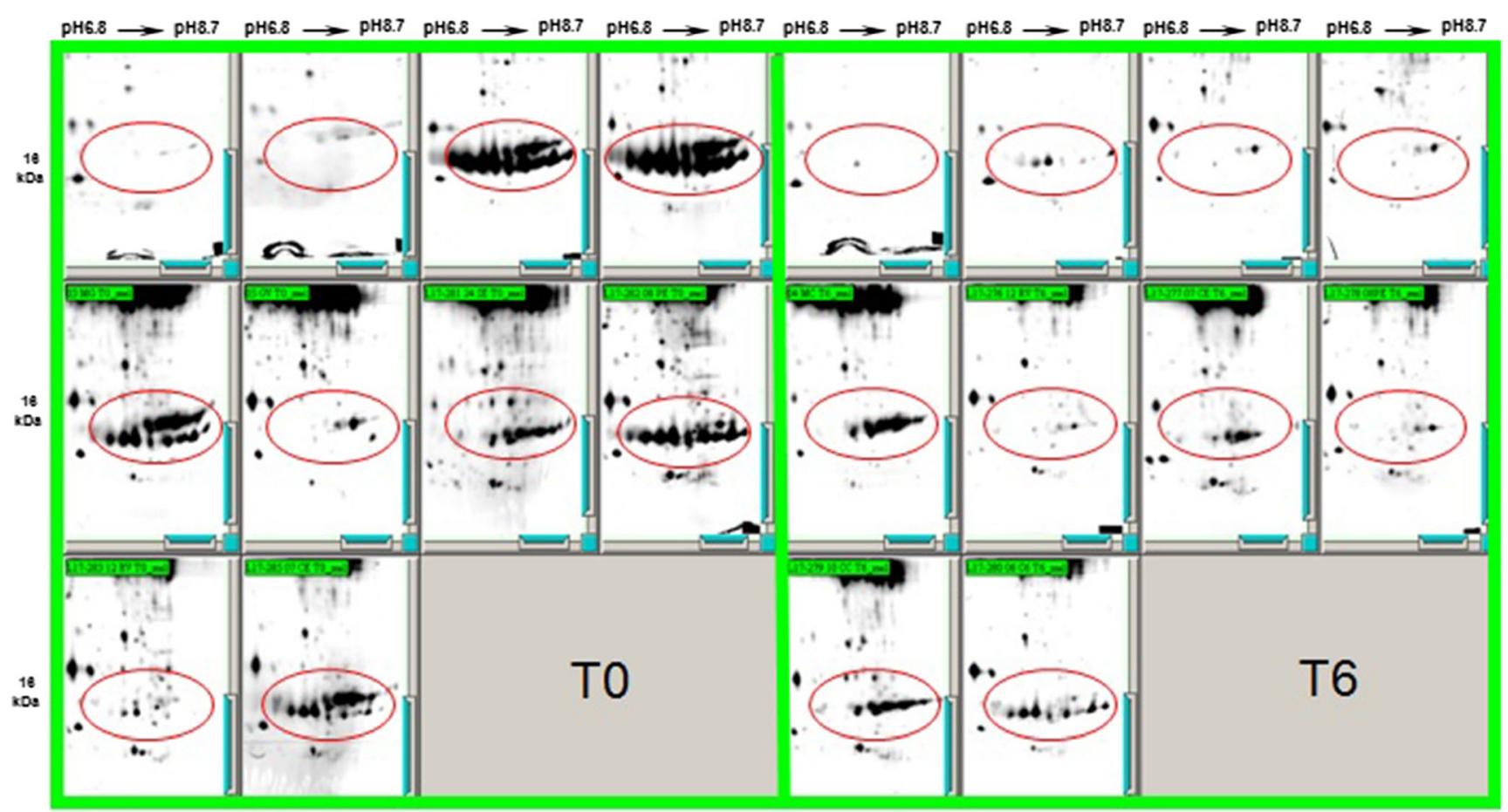

Fig. 2 Differential proteomic analysis in T0 and T6 conditions. Highlights of basic and low molecular weight portions in all obtained gels, bringing to light highly present spots in $\mathrm{T} 0$ (on the left part) with respect to the T6 ones (right part) circled in red

\section{Enrichment analysis by STRING software}

STRING analysis was performed in order to elaborate an enrichment analysis, summarizing functional information about the identified proteins with respect to the three Gene Ontology categories of biological process, molecular function, and cellular component and to predict possible associations among them. STRING networks consist of nodes (spheres) representing proteins, while edges represent the predicted mode of action among proteins (Fig. 5). The analysis was elaborated in order to connect our protein species with a maximum of five more interactors. In both networks obtained and shown in Fig. 5, it is possible to observe the identified proteins connected with the predicted functional partners such as for coagulator factor II (thrombin, F2), transferrin receptor (TFRC), HLA-A, and HLA-B. The three colors of the spheres in Fig. 5a (green, red, and blue) identify the three clusters of functionally correlated proteins. In Fig. 5b, the different colored spheres are correlated with their respective biological processes. HBA1, HBA2, HBB, HP, ALB, and transferrin receptor (red wedge) correlate with the "receptor-mediated endocytosis" Gene Ontology term; B2M, HLA-A, and HLA-B (blue wedge) correlate with "antigen processing and presentation of exogenous peptide antigen via MHC class I, TAP-independent"; HBA1, HBA2, HBB, HP, TFRC, and $\mathrm{B} 2 \mathrm{M}$ (green wedge) are related to "response to inorganic substance"; HBA1, HBA2, HBB (pink wedge) to "oxygen transport" and almost all of them (HBA1, HBA2, HBB, HP, TFRC, B2M, ALB, HLA-A, HLA-B, F2, and SERPINC1) (yellow wedge) to "response to stress." About molecular functions and cellular components enriched terms, interestingly, among the KEGG pathways (Kyoto Encyclopedia of Genes and Genomes), we found "antigen processing and presentation" (HLA-A, HLA-B and B2M), "endocytosis" (HLA-A, HLA-B, and TFRC), and "phagosome" (HLA-A, HLA-B, and TFRC) pathways, which were strongly suggested by the proteins identified in this study.

\section{Discussion}

In this study, we explored four different parameters, namely neurological condition, CSF differential proteome, inflammatory cytokines, and activated T cells before and after a specific multistrain probiotic supplementation. From a clinical point of view, we confirmed the improvement of neurological impairment observed in a group of HIV patients during cART and with undetectable levels of HIV viremia after probiotic supplementation with Vivomixx ${ }^{\circledR}$ (Ceccarelli et al. 2017a). Our results confirmed that supplementing cART with a multistrain probiotic might favor the composition of commensal gut microbiota with immune restorative process and a return to physiological status as demonstrated in previous studies (d'Ettorre et al. 2017).

The supplementation of cART with oral bacteriotherapy was also reflected in the CSF proteome of these patients. In particular, our results suggest that probiotic treatment modifies protein species composition and abundance at the CSF level. 


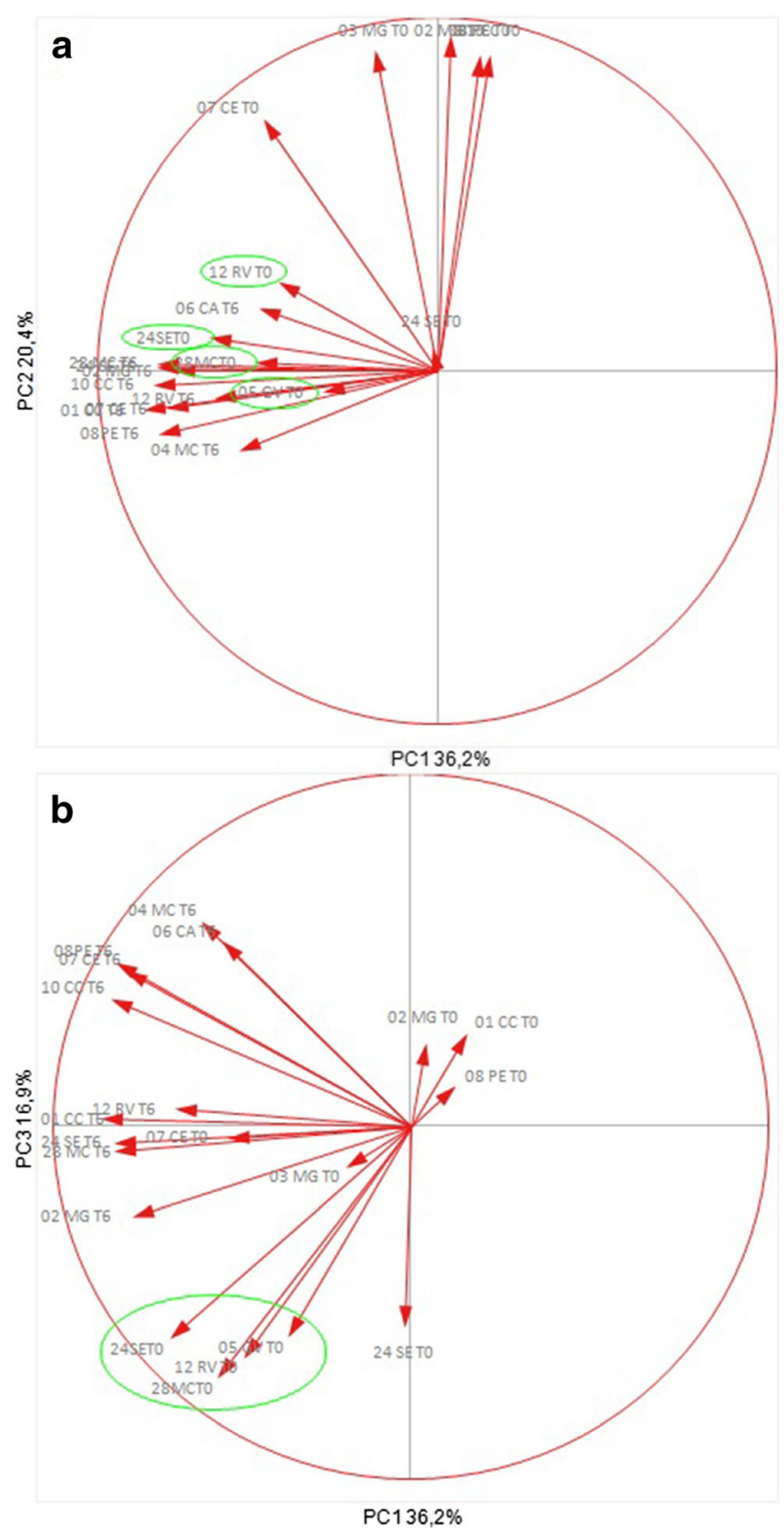

Fig. 3 T0 vs T6 principal component analysis. PCA was performed on $\% \mathrm{~V}$ of the differentially abundant spots. The graph A highlights the spatial distribution of the $20 \mathrm{CSF}$ samples along the PC1 and PC2 while the graph B shows the distribution of the 20 samples along the $\mathrm{PC} 1$ and $\mathrm{PC} 3$ in order to evaluate the samples distribution on threedimensional plane. Green circles highlight 4 T0 samples with an independent behavior from the rest of the $\mathrm{T} 0$ group

Interestingly, T6 samples evidenced in PCA a lower distance correlation in a well-defined area with respect to T0 samples, indicating a differential protein pattern profile with more homogeneous behavior and suggesting a re-establishment of the protein composition and abundance in the CSF after 6 months of treatment. The prevailing reduction of several inflammatory proteins after 6 months of probiotic supplementation suggests an amelioration of the CNS inflammatory state.

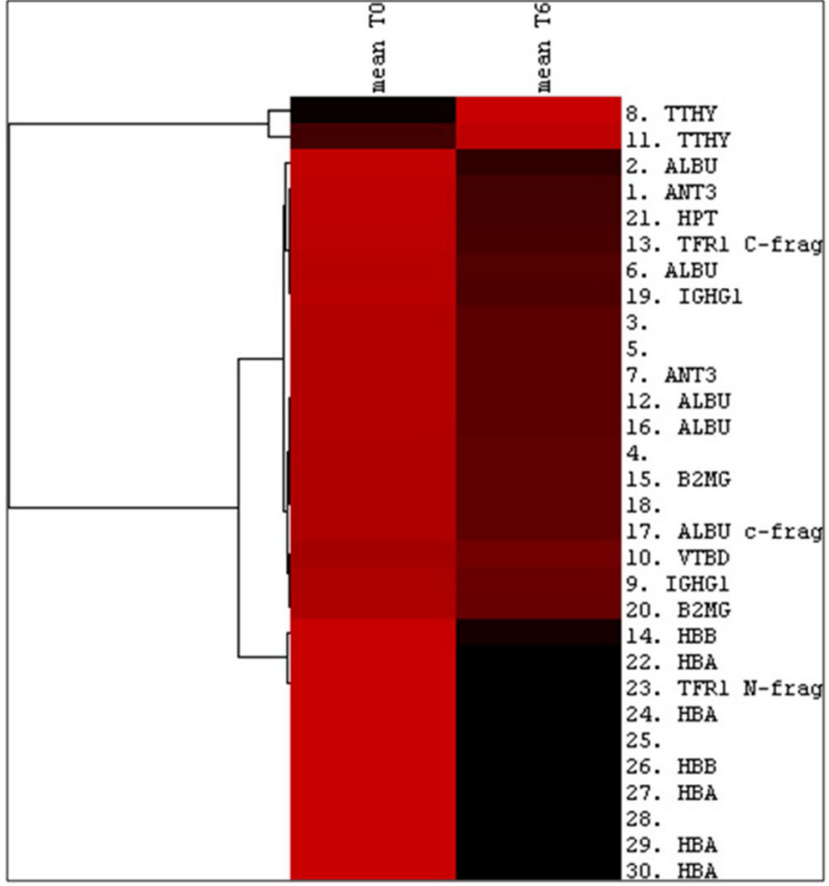

Fig. 4 Cluster analysis. Normalized values of the $\% \mathrm{~V}$ means of the differentially abundant spots (number and identified protein name on the right) were reported as colored portions. Higher values correspond to red. Lower values correspond to black. The hierarchical tree characterizing two main clusters of proteins with similar behavior is reported on the left

Interestingly, only two spots which corresponded to transthyretin (TTR) increased at T6, in agreement with previous data showing an inverse relationship between TTR levels in the CSF and neurological inflammation (Ambalavanan et al. 2005). Moreover, TTR is important for thyroxine (T4) transfer from blood to CSF through the blood-brain barrier (BBB) and protects the brain from $\beta$-amyloid neurotoxic accumulations into amyloid plaques, commonly observed in the early stages of Alzheimer's disease and other disorders such as schizophrenia (Chen et al. 2016; Richardson et al. 2015). Among the abundant proteins at $\mathrm{T} 0$, we observed different protein species of albumin, consistent with Rahimy et al. (2017) and also spots of hemoglobin $\alpha$ and $\beta$, which is physiologically absent, or present at very low levels, in healthy CSF. These results strongly suggest the presence of a BBB impairment, characterized by the loss of its selective competence to regulate molecule exchange between blood vessels and the CSF, independently of disease stadiation. After 6 months of probiotic administration, the homogeneous decrease in abundance of the differential proteins suggests a possible recovery of the protective action mechanism of the BBB. Furthermore, since our CSF samples were not contaminated by blood cells during the spinal tap procedure, the marked decrease or disappearance of hemoglobin spots at T6 confirms that probiotic treatment was followed by an improvement in BBB permeability. 
Fig. 5 Protein net by STRING analysis (a). Number of nodes are 9 , number of edges are 24, average node degree is 5.33 , avg. local clustering coefficient is 0.731 , expected number of edges are 2, and PPI enrichment $p$ value is $<1.0 \mathrm{e}-16$. The colors (light blue, red, and green) represent the three different clusters of the proteins in the net. $\mathbf{b}$ Colors on the sphere represent proteins correlated to a specific biological process as reported
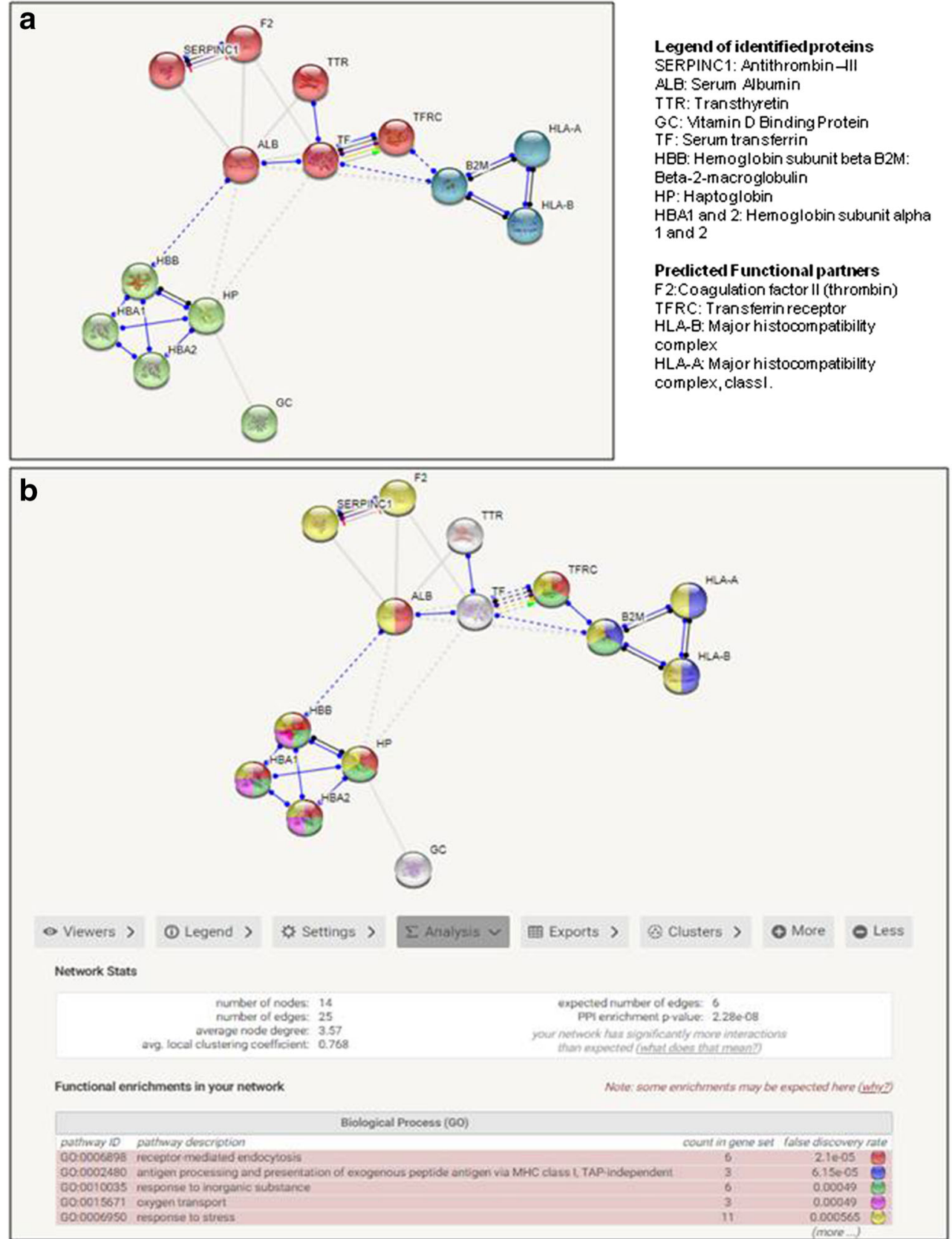

This modification has a functional impact since products derived from hemoglobin metabolism greatly contribute to brain injury. For instance, iron promotes redox imbalance by mediating free radical generation, amplifies inflammatory response, and prevents DNA repair (Righy et al. 2018). Our results are also supported by the decreased-abundance of haptoglobin and N-terminal and C-terminal fragments of transferrin in $8 \mathrm{CSF}$ samples at T6 as compared to T0. Haptoglobin is a plasma protein synthesized by the liver that functions by binding free hemoglobin and heme released during intravascular hemolysis, thereby removing them from circulation (Righy et al. 2018). Its decrease at
T6 therefore suggests a decreased level of inflammation. The high abundance of $\mathrm{N}$-terminal and $\mathrm{C}$-terminal fragments of transferrin in the CSF at T0, which tend to be reduced after 6 months of treatment, suggests that fragmentation of transferrin in cART patients plays a role in the dysfunction of the iron transport system and in its cerebral homeostasis. Mehta et al. observed an abnormal iron homeostasis in HIV neurocognitive impairment patients due to a dysregulation of iron transport in the brain (Mehta et al. 2017). The use of probiotics seems to restore the physiological condition wherein transferrin is not degraded, therefore maintaining its protective function. 
According to the KEGG pathway, the differentially abundant proteins are mainly involved in pathways corresponding to antigen processing and presentation, endocytosis, and phagosome. Of note, our study indicates that beta-2microglobulin (B2M) is particularly involved in these processes, considering that its CSF levels are directly related to the presence of inflammatory, autoimmune, or neoplastic diseases in the CNS and to disease progression in HIV patients (Svatoňová et al. 2014). Indeed, B2M constitutes the light chain of the main proteins of the MHC I complex and increased values of B2M in CSF are found to be a sign of immune activation, abnormal turnover of lymphoid cells, and tissue damage in the CNS. A decreased amount of B2M following probiotic administration indicates an improvement in the immune status of the patient and a reduction in their inflammatory condition.

The novelty of this study is that it was able to evidence an amelioration of the CNS inflammatory state due to improved BBB permeability in the CSF of patients who experienced an improvement of their neuropsychiatric function. Surprisingly, based on prior literature suggesting potential alterations of blood-CSF permeability in HIV subjects, we were expecting that clinical and CSF improvement would be accompanied by a reduction in the abnormally high pro-inflammatory plasma cytokine levels. However, this was not observed in our patients, regardless of disease stage. The neurological condition improvement at $\mathrm{T} 6$ was not accompanied by any improvement in the pro-inflammatory cytokines that we measured, including IL-6, sCD14, and MIP-1 $\beta$. Nevertheless, we cannot rule out that the power of statistical results has been reduced by the small sample size studied.

Indeed, a significant limitation of our study was the small sample size which consisted of only 13 patients. However, it was necessary to limit the sample size due to the invasive nature of performing a lumbar puncture, which has inherent risks. Additionally, the cost per patient of conducting this study was $\$ 10,000$, which further impacted the sample size. Another limitation is that the interpretation of scores on batteries of neurocognitive tests repeated over several administrations can be complicated by the so-called practice effect: in fact, this improvement can result from patient's learning across multiple test administrations. To limit this potential risk, we chose to use alternate and parallel forms designed to avoid or reduce content- or item-specific practice effects that can be associated with repeated administrations of the same neuropsychological tests, on the basis of previous published evidences (Ceccarelli et al. 2017a, b; Getz 2011; Ferguson and Iverson 2011; Benedict and Zgaljardic 1998; Benedict 2005; Pereira et al. 2015).

Due to the limited number of patients enrolled, we were not able to offer any correlation between the various parameters studied. However, some data are so homogeneous that some conclusions can be drawn and form the basis for larger studies in the future. For example, while the improvement of neuropsychiatric conditions in cART patients has been confirmed in this study as well as in previous studies, the mechanism by which probiotic supplementation leads to changes in brain function is not elucidated. Among the multiple hypotheses advanced, the most accepted ones involve a probioticinduced change of the gut flora metabolites or of gut permeability, and decreased systemic production of proinflammatory cytokines, with a special emphasis on TNF- $\alpha$.

Additionally, the clinical improvement, amelioration of the CSF proteomic profile, and reduction in the circulating activated mononuclear cells without any modification of proinflammatory cytokines leads us to hypothesize that the use of specific multistrain probiotic may also exert a local protective mechanism at the level of the choroid plexus. In nonpathological conditions, the choroid plexus is involved in immunosurveillance and interfaces between the CNS and peripheral circulation, in addition to its role in CSF production. In a similar way, it has been demonstrated that probiotics act at the level of the tight junctions in the gut (d'Ettorre et al. 2017). Further studies should now investigate if the tight junctions of the choroid plexus can also be protected.

Acknowledgments This study was partially supported by Mendes s.r.l. to Bini Luca.

Data availability statement In accord to Italian law and regulations on patients' privacy, data are not publicly available but can eventually be made available upon request.

Author's contribution C.L.: Design and conceptualized the study, analyzed the data, and drafted the manuscript for intellectual content. L.S., L.B., and S.E.: Acquired, analyzed, and interpreted the data. G.C.: Enrolled patients, analyzed and interpreted the data, and drafted and revised the manuscript for intellectual content. ENC Enrolled patients, critically revised data and manuscript. CB: critically revised data and performed and revised statistical analysis. C.P.: Acquired, analyzed, and interpreted the data. C.S.: Acquired, analyzed, and interpreted the data, drafted the manuscript for intellectual content. V.V., L.B, and G.d'E.: design and conceptualized study, analyzed the data, drafted the manuscript for intellectual content.

\section{Compliance with ethical standards}

The study was approved by the internal committee of the Infectious Diseases Department and by the Ethics Committee of "Sapienza" University of Rome. A written informed consent was obtained from all patients prior to enrollment.

Conflict of interest Dr. Landi, Dr. Santinelli, Dr. Bianchi, Dr. Shaba, Dr. Ceccarelli, Dr. Pinacchio, Prof. Scagnolari, Prof. Vullo, and Prof. d'Ettorre report no disclosures. Prof. Bini was partially supported by Mendes s.r.l. (Ardea, Roma).

\section{References}

Akbari E, Akbari E, Asemi Z, Daneshvar Kakhaki R, Bahmani F, Kouchaki E, Tamtaji OR, Hamidi GA, Salami M (2016) Effect of 
probiotic supplementation on cognitive function and metabolic status in Alzheimer's disease: a randomized, double-blind and controlled trial. Front Aging Neurosci 8:256. https://doi.org/10.3389/ fnagi.2016.00256

Ambalavanan N, Ross AC, Carlo WA (2005) Retinol-binding protein, transthyretin, and C-reactive protein in extremely low birth weight (ELBW) infants. J Perinatol 25(11):714-719. https://doi.org/10. 1038/sj.jp. 7211398

Banks WA, Gray AM, Erickson MA, Salameh TS, Damodarasamy M, Sheibani N, Meabon JS, Wing EE, Morofuji Y, Cook DG, Reed MJ (2015) Lipopolysaccharide-induced blood-brain barrier disruption: roles of cyclooxygenase, oxidative stress, neuroinflammation, and elements of the neurovascular unit. J Neuroinflammation 12:223. https://doi.org/10.1186/s12974-015-0434-1

Benedict R (2005) Effects of using same- versus alternate-form memory tests during short-interval repeated assessments in multiple sclerosis. J Int Neuropsychol Soc 11(6):727-736. https://doi.org/10.1017/ S1355617705050782

Benedict RH, Zgaljardic DJ (1998) Practice effects during repeated administrations of memory tests with and without alternate forms. J Clin Exp Neuropsychol 20(3):339-352

Bini L, Magi B, Cellesi C, Rossolini A, Pallini V (1992) Twodimensional electrophoresis analysis of human serum proteins during the acute-phase response. Electrophoresis 13(9-10):743-746

Bini L, Magi B, Marzocchi B, Cellesi C, Berti B, Raggiaschi R, Rossolini A, Pallini V (1996) Two-dimensional electrophoresis patterns of acute phase human serum proteins in the course of bacterial and viral diseases. Electrophoresis 17:612-616

Carter GM, Esmaeili A, Shah H, Indyk D, Johnson M, Andreae M, Sacks HS (2016) Probiotics in human immunodeficiency virus infection: a systematic review and evidence synthesis of benefits and risks. Open Forum Infect 3:ofw164. https://doi.org/10.1093/ofid/ofw164

Ceccarelli G, Brenchley JM, Cavallari EN, Scheri GC, Fratino M, Pinacchio C, Schietroma I, Fard SN, Scagnolari C, Mezzaroma I, Vullo V, d'Ettorre G (2017a) Impact of high-dose multi-strain probiotic supplementation on neurocognitive performance and central nervous system immune activation of HIV-1 infected individuals. Nutrients 9(11). https://doi.org/10.3390/nu9111269

Ceccarelli G, Fratino M, Selvaggi C, Giustini N, Serafino S, Schietroma I, Corano Scheri G, Pavone P, Passavanti G, Alunni Fegatelli D, Mezzaroma I, Antonelli G, Vullo V, Scagnolari C, d'Ettorre G (2017b) A pilot study on the effects of probiotic supplementation on neuropsychological performance and microRNA-29a-c levels in antiretroviral-treated HIV-1-infected patients. Brain Behav 7: e00756. https://doi.org/10.1002/brb3.756

Chen JJ, Genereux JC, Suh EH, Vartabedian VF, Rius B, Qu S, Dendle MTA, Kelly JW, Wiseman RL (2016) Endoplasmic reticulum Proteostasis influences the oligomeric state of an Amyloidogenic protein secreted from mammalian cells. Cell Chem Biol 23(10): 1282-1293. https://doi.org/10.1016/j.chembiol.2016.09.001

Cinque P, Brew BJ, Gisslen M, Hagberg L, Price RW (2007) Cerebrospinal fluid markers in central nervous system HIV infection and AIDS dementia complex. In: Vinken PJ, Bruyn GW (eds) Handbook of clinical neurology, vol 85, pp 261-300

D'Angelo C, Reale M, Costantini E (2017) Microbiota and probiotics in health and HIV infection. Nutrients 9:615. https://doi.org/10.3390/ nu9060615

d'Ettorre G, Baroncelli S, Micci L, Ceccarelli G, Andreotti M, Sharma P, Fanello G, Fiocca F, Cavallari EN, Giustini N, Mallano A, Galluzzo CM, Vella S, Mastroianni CM, Silvestri G, Paiardini M, Vullo V (2014) Reconstitution of intestinal CD4 and Th17 T cells in antiretroviral therapy suppressed HIV-infected subjects: implication for residual immune activation from the results of a clinical trial. PlosOne. 9(10):e109791. https://doi.org/10.1371/journal.pone. 0109791
d'Ettorre G, Rossi G, Scagnolari C, Andreotti M, Giustini N, Serafino S, Schietroma I, Scheri GC, Fard SN, Trinchieri V, Mastromarino P, Selvaggi C, Scarpona S, Fanello G, Fiocca F, Ceccarelli G, Antonelli G, Brenchley JM, Vullo V (2017) Probiotic supplementation promotes a reduction in T-cell activation, an increase in Th17 frequencies, and a recovery of intestinal epithelium integrity and mitochondrial morphology in ART-treated HIV-1-positive patients. Immun Inflamm Dis 5(3):244-260. https://doi.org/10.1002/iid3.160

Ferguson KE, Iverson GL (2011) Alternate test forms. In: Kreutzer JS, DeLuca J, Caplan B (eds) Encyclopedia of clinical neuropsychology. Springer, New York

Getz GE (2011) Parallel Forms. In: Kreutzer JS, DeLuca J, Caplan B (eds) Encyclopedia of clinical neuropsychology. Springer, New York

Gold JA, Grill M, Peterson J, Pilcher C, Lee E, Hecht FM, Fuchs D, Yiannoutsos CT, Price RW, Robertson K, Spudich S (2014) Longitudinal characterization of depression and mood states beginning in primary HIV infection. AIDS Behav 18(6):1124-1132. https://doi.org/10.1007/s10461-013-0688-5

Heaton RK, Clifford DB, Franklin DR Jr, Woods SP, Ake C, Vaida F, Ellis RJ, Letendre SL, Marcotte TD, Atkinson JH, Rivera-Mindt M, Vigil OR, Taylor MJ, Collier AC, Marra CM, Gelman BB, McArthur JC, Morgello S, Simpson DM, McCutchan JA, Abramson I, Gamst A, Fennema-Notestine C, Jernigan TL, Wong J, Grant I, CHARTER Group (2010) HIV-associated neurocognitive disorders persist in the era of potent antiretroviral therapy: CHARTER study. Neurology 75(23):2087-2096. https://doi.org/ 10.1212/WNL.0b013e318200d727

Heaton RK, Franklin DR, Ellis RJ, McCutchan JA, Letendre SL, Leblanc S, Corkran SH, Duarte NA, Clifford DB, Woods SP, Collier AC, Marra CM, Morgello S, Mindt MR, Taylor MJ, Marcotte TD, Atkinson JH, Wolfson T, Gelman BB, McArthur JC, Simpson DM, Abramson I, Gamst A, Fennema-Notestine C, Jernigan TL, Wong J, Grant I, CHARTER Group; HNRC Group (2011) HIVassociated neurocognitive disorders before and during the era of combination antiretroviral therapy: differences in rates, nature, and predictors. J Neuro-Oncol 17(1):3-16. https://doi.org/10.1007/ s13365-010-0006-1

Hochstrasser DF, Patchornik A, Merril CR (1988) Development of polyacrylamide gels that improve the separation of proteins and their detection by silver staining. Anal Biochem 173:412-423

Hunt PW, Sinclair E, Rodriguez B, Shive C, Clagett B, Funderburg N, Robinson J, Huang Y, Epling L, Martin JN, Deeks SG, Meinert CL, Van Natta ML, Jabs DA, Lederman MM (2014) Gut epithelial barrier dysfunction and innate immune activation predict mortality in treated HIV infection. J Infect Dis 210:1228-1238. https://doi.org/ 10.1093/infdis/jiu238

Mehta SR, Pérez-Santiago J, Hulgan T, Day TR, Barnholtz-Sloan J, Gittleman H, Letendre S, Ellis R, Heaton R, Patton S, Suben JD, Franklin D, Rosario D, Clifford DB, Collier AC, Marra CM, Gelman BB, McArthur J, McCutchan A, Morgello S, Simpson D, Connor J, Grant I, Kallianpur A (2017) Cerebrospinal fluid cell-free mitochondrial DNA is associated with HIV replication, iron transport, and mild HIV-associated neurocognitive impairment. J Neuroinflammation 14(1):72. https://doi.org/10.1186/s12974-0170848-z

Pereira DR, Costa P, Cerqueira JJ (2015 Aug) Repeated assessment and practice effects of the written symbol digit modalities test using a short inter-test interval. Arch Clin Neuropsychol 30(5):424-434. https://doi.org/10.1093/arclin/acv028

Pinacchio C, Scheri GC, Statzu M, Santinelli L, Ceccarelli G, Innocenti GP et al (2018) Type I/II interferon in HIV-1-infected patients: expression in gut mucosa and in peripheral blood mononuclear cells and its modification upon probiotic supplementation. J Immunol Res 2018:1738676 
Rahimy E, Li FY, Hagberg L, Fuchs D, Robertson K, Meyerhoff DJ, Zetterberg H, Price RW, Gisslén M, Spudich S (2017) Blood-brain barrier disruption is initiated during primary HIV infection and not rapidly altered by antiretroviral therapy. J Infect Dis 215(7):11321140. https://doi.org/10.1093/infdis/jix013

Richardson SJ, Wijayagunaratne RC, D'Souza DG, Darras VM, Van Herck SL (2015) Transport of thyroid hormones via the choroid plexus into the brain: the roles of transthyretin and thyroid hormone transmembrane transporters. Front Neurosci 9:66. https://doi.org/10. 3389/fnins.2015.00066

Righy C, Turon R, Freitas G, Japiassú AM, Faria Neto HCC, Bozza M, Oliveira MF, Bozza FA (2018) Hemoglobin metabolism byproducts are associated with an inflammatory response in patients with hemorrhagic stroke. Rev Bras Ter Intensiva 30(1):21-27. https://doi.org/10.5935/0103-507x.20180003

Salas JT, Chang TL (2014) Microbiome in human immunodeficiency virus infection. Clin Lab Med 34:733-745. https://doi.org/10. 1016/j.cll.2014.08.005

Spudich SS, Spudich SS, Nilsson AC, Lollo ND, Liegler TJ, Petropoulos CJ, Deeks SG, Paxinos EE, Price RW (2005) Cerebrospinal fluid HIV infection and pleocytosis: relation to systemic infection and antiretroviral treatment. BMC Infect Dis 5:98. https://doi.org/10. 1186/1471-2334-5-98

Spudich S, Gisslen M, Hagberg L, Lee E, Liegler T, Brew B, Fuchs D, Tambussi G, Cinque P, Hecht FM, Price RW (2011) Central nervous system immune activation characterizes primary human immunodeficiency virus 1 infection even in participants with minimal cerebrospinal fluid viral burden. J Infect Dis 204(5):753-760. https://doi. org/10.1093/infdis/jir387

Svatoňová J, Bořecká K, Adam P, Lánská V (2014) Beta2-microglobulin as a diagnostic marker in cerebrospinal fluid: a follow-up study. Dis Markers 2014:495402-495406. https://doi.org/10.1155/2014/ 495402

Vera JH, Guo Q, Cole J, Boasso A, Greathead L, Kelleher P, Rabiner I, Bishop C, Matthews P, Gunn R, Winston A (2015) Microbial translocation is associated with Neuroinflammation in HIV subjects on ART. CROI Conference abstract 477 (session P-G6)

Vera JH, Guo Q, Cole JH, Boasso A, Greathead L, Kelleher P, Rabiner EA, Kalk N, Bishop C, Gunn RN, Matthews PM, Winston A (2016) Neuroinflammation in treated HIV-positive individuals: a TSPO PET study. Neurology 86(15):1425-1432. https://doi.org/10.1212/ WNL.0000000000002485

Xu H, Wang X, Veazey RS (2013) Mucosal immunology of HIV infection. Immunol Rev 254:10-33. https://doi.org/10.1111/imr.12072

Publisher's note Springer Nature remains neutral with regard to jurisdictional claims in published maps and institutional affiliations. 